\title{
DEVELOPMENT OF HIGH PERFORMANCE PASSIVELY COOLED MIRRORS FOR ECH LAUNCHERS
}

\author{
Princeton Plasma Physics Laboratory \\ Princeton, NJ 08543-0451 USA \\ rellis@pppl.gov
}

\author{
H. Grunloh, J. Lohr \\ General Atomics \\ San Diego, CA 92121
}

\begin{abstract}
Fusion reactors and state-of-the-art tokamaks will operate at pulse lengths that require heat absorbing components to be designed for steady state operation. Electron Cyclotron Heating (ECH) power sources are approaching that goal as well. The reflecting mirrors for ECH launchers will therefore need to be designed for steady-state operation.
\end{abstract}

There are still a number of ECH systems that do not operate at steady state, but at increasing pulse lengths and power levels. At these facilities, despite some advantages that would result from using actively cooled ECH launcher mirrors, operating costs and risks can be minimized by using passively cooled mirrors that are designed to be replaced with a minimum of difficulty.

This paper describes the design of the most recent mirrors for the DIII-D ECH launchers. Electromagnetic and thermal stress analyses are presented, and the fatigue life of the mirrors is estimated. The costs and benefits of periodic replacement of mirrors, as compared to the use of steady-state actively cooled mirrors, are discussed.

\section{INTRODUCTION}

Electron Cyclotron Heating has emerged as a critical element in advanced tokamaks ${ }^{1,2,3}$, both for plasma heating and, more recently, for stabilizing neoclassical tearing modes using Electron Cyclotron Current Drive (ECCD) ${ }^{4}$. In either ECH or ECCD, a beam, typically $\sim 6 \mathrm{~cm}$ diameter, is launched into a tokamak vacuum vessel by reflecting it off a fixed mirror, which may provide some focusing, and then off a steerable mirror. Typically, between $0.15 \%$ and $0.2 \%$ of incident power is absorbed by each mirror. The frequency of the EC beam is determined by matching the electron cyclotron resonant frequency, or one of its harmonics, to the frequency of a commercially available gyrotron. The DIII-D ECH system ${ }^{5}$ uses $110 \mathrm{GHz}$ to match the second harmonic. KSTAR uses 84 $\mathrm{GHz}$ for its initial EC system to match the first harmonic, and will use $170 \mathrm{GHz}$ second harmonic operation for its second system, in order to be relevant to ITER.

Many existing tokamaks and their associated heating systems operate at pulse lengths less than ten seconds. For ECH pulse lengths of up to 5 seconds and beam power levels up to $\sim 1 \mathrm{MW}$, it has been acceptable to rely on passive, or inertial, cooling of the mirrors. The DIII-D ECH system has progressed to this level in recent years, and a new system will extend this power and pulse length to consistently operate at
$1.5 \mathrm{MW}$ for 10 seconds, with pulses every 15 minutes.

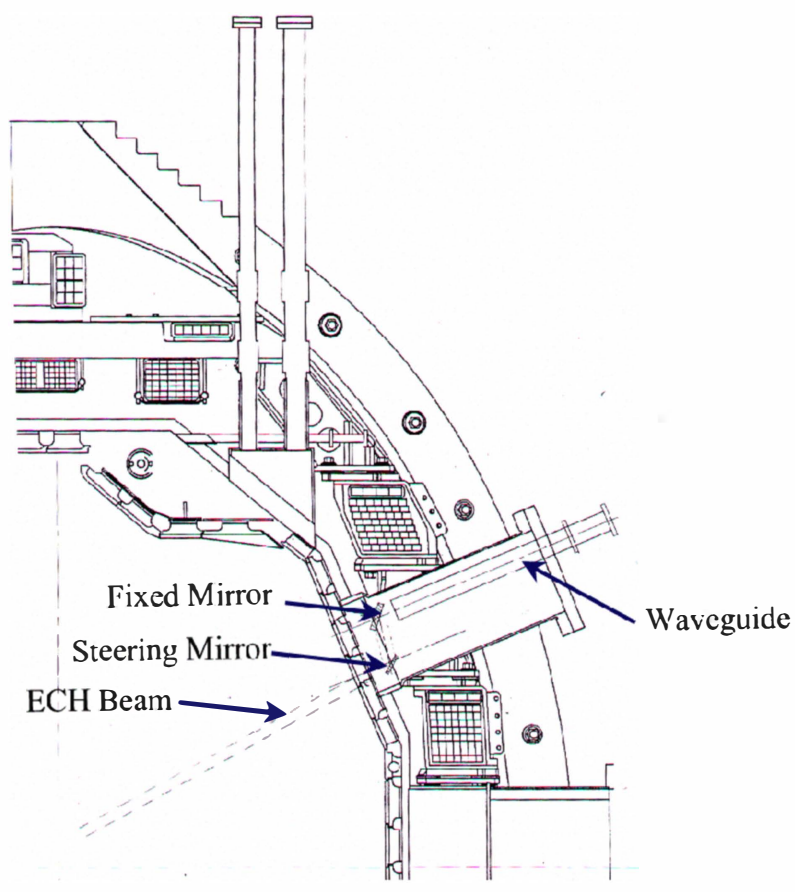

Figure 1. Schematic Launcher Elevation

The ECH launchers currently in operation on DIII-D have been in service since the installation of the P2001 launcher ${ }^{6}$ in 2001. Since then, we have added the P2002 and P2006 launchers. During this time, we have steadily improved the design of the fixed mirrors, while the steerable mirrors originally supplied in 2001 have remained adequate. The new P2012 ECH launcher is expected to operate with the $1.5 \mathrm{MW}$ power source, and we therefore improved the designs of the fixed and steerable mirrors. The KSTAR ECH launchers share many design elements with the DIII-D launchers, and the mirror development is relevant to both.

During the design of the new mirrors, both actively cooled, steady-state mirrors and passively cooled mirrors were considered. While it is evident that the passively cooled mirrors, because of their necessarily large temperature excursions, will experience significant thermal fatigue stresses and therefore require periodic replacement, the lower 
operational and developmental risk made them a good choice for this application. This paper discusses the evolution of the design of the fixed mirrors for the P20xx DIII-D launchers, and the design of the new steerable mirrors.

\section{FIXED MIRROR DESIGN EVOLUTION}

The fixed mirrors of the ECH launchers provide mild focusing of the incoming beam, and direct the beam onto the steerable mirrors. Some adjustability in the mirror mounting is required for alignment of the beam. These mirrors rely on large temperature increases and a significant heat capacity to absorb the power from the incident beam. This heat is then radiated and conducted out of the mirror during the time between pulses.

Electromagnetic forces result from plasma disruptions, during which the rate of flux change at the launcher is 200 Tesla/second. In DIII-D, the ambient toroidal field is $1.5 \mathrm{~T}$ at the location of the launcher.

Thermal loads due to the ECH beam are highly peaked, decreasing as the square of the Bessel function of distance from the beam axis. The design of the mirror is dictated by the heat flux distribution, as well as the electromagnetic forces: make the center of the mirror thick in order to diffuse heat, and make the edges thin in order to minimize eddy currents.

\section{A. P2001 Fixed Mirrors}

The original fixed mirrors on the P2001 launcher followed this approach, with some compromises for structural integrity, ease of fabrication, and alignment. The mirrors were a threepart brazed assembly - a stainless steel reflecting plate with the parabolic contour machined in, a copper hemisphere to diffuse heat and provide for angular adjustment, and a stainless steel stud to hold the mirror assembly together. A recess is machined into the stainless steel plate for the mounting ball, so that there is a minimal, .02 " $[0.5 \mathrm{~mm}]$ thickness of stainless steel between the reflecting surface and the hemisphere. The front surface of the stainless steel plate is coated with less than 0.002 " $[0.05 \mathrm{~mm}]$ of copper. Holes were drilled from the outside of the hemisphere through the center of the mounting stud so that two resistive temperature detectors [RTD] could be installed.

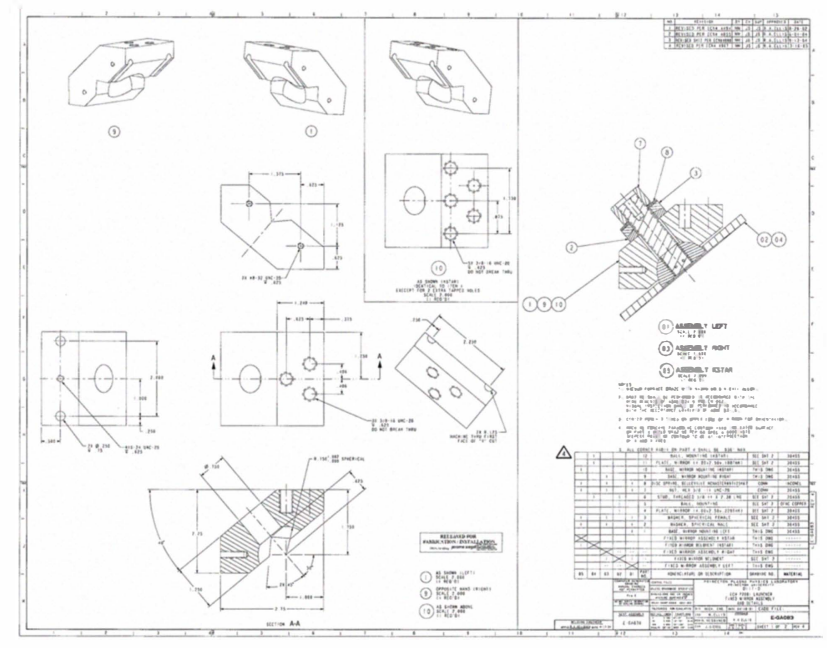

Figure 2. P2001 Fixed Mirror and Mount Assembly

\section{B. P2006 Fixed Mirrors}

After initial operations with the P2001 launcher, and then the P2002 launcher, we noticed some dimpling near the center of the fixed mirrors. The stainless steel stud did not diffuse heat adequately, and the front surface temperature at the mirror center was excessive. For the P2006 launcher, we modified this stud to include a copper plug, .313" diameter and .5" deep, at the end in contact with the reflecting plate. The heat would diffuse rapidly into the copper plug, and into the copper hemisphere surrounding the threaded stud. Thermal diffusion through the stainless steel between the copper plug and the hemisphere would also occur rapidly because of the thin cross section. Fixed mirrors of this design were also installed on the P2001 and P2002 launchers.

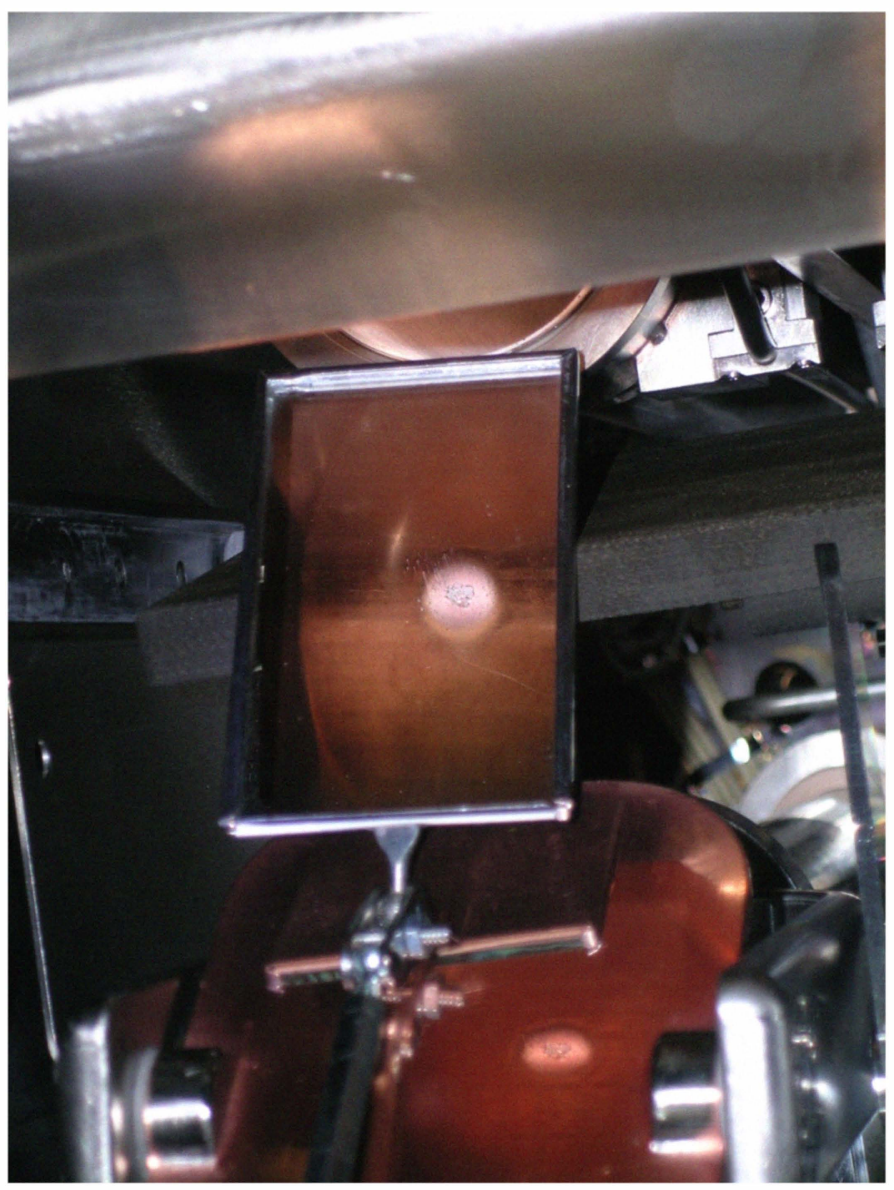

Figure 3. Incipient Thermal Fatigue on Fixed Mirror

As power levels and pulse lengths increased, we observed some dimpling at the center of the mirror, in an annular pattern around a clean section at the center of the mirror. Eventually, the stainless steel below the plating was exposed to the incident beam. Because of its high resistivity, stainless steel experiences a heat flux roughly seven times that of copper. Deterioration of the copper plating leads rapidly to catastrophic failure of the mirror.

Finite element thermal and stress analysis of these mirrors showed a pattern of stresses matching the ring pattern of 
dimpling of the front surface of the mirror. We concluded that thermal fatigue was the cause of the mirror failure.

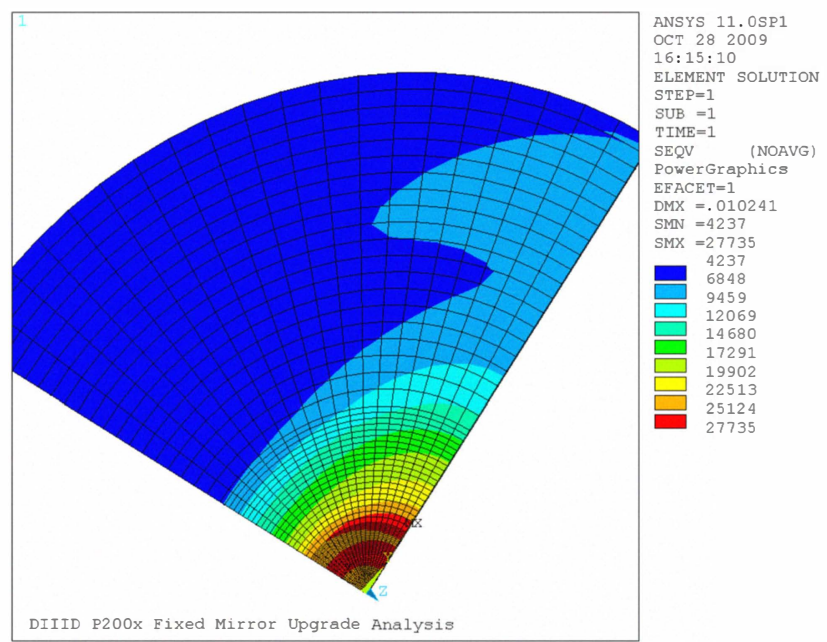

Figure 4. Finite Element Analysis of P2006 Fixed Mirror

\section{Fixed Mirrors - 2008 Upgrade}

In 2008, we designed another upgrade of the fixed mirrors. The copper plated stainless steel reflecting plate was the same as before, but we now made the mounting stud from $\mathrm{Cu}-\mathrm{Cr}$ [C18200]. In addition, the holes for the RTDs no longer passed through the center of the mounting stud, as this produced a void a short distance behind the front surface. Three of the six launcher mirrors were replaced with this version, two in 2008 and one in 2009, and they operated through the 2010 experimental campaign without incident.

\section{P2012 ECH Launcher Fixed Mirrors}

The newest ECH launcher for DIII-D, scheduled for delivery in 2011, will be connected to a gyrotron capable of $1.5 \mathrm{MW}$ pulses for 10 second durations. This increased power dictated new designs for the fixed and steerable mirrors.

A one-piece fixed mirror, made from a copper alloy, was considered for the new fixed mirrors. Our ability to analyze electromagnetic forces has improved significantly since the original mirrors were designed, and we felt that the more accurately analyzed forces in a one-piece mirror would be within the conservative design allowable from the original mirrors.

We also wanted to retain as much as possible of the original mounting system, so that we would maximize the compatibility of the new mirrors with the existing launchers. The initial concept retained the hemisphere and threaded rod of the existing design, although they would now be turned out of one block of metal, and a reflecting plate of constant thickness. Electromagnetic analysis of currents and forces due to a disruption indicated that the mounting stud and hemisphere would be adequate for the DIII-D launcher, but that a stronger bolt pattern would be required. This design would not be adequate for KSTAR due to the higher toroidal field on that machine.
The design was refined by reducing the thickness of the reflecting plate away from the center of the mirror. Because of the peaked heat load, the mirror does not need to be as thick towards the edges. This change reduced the magnitude of the eddy currents, and also pushed the current path closer to the center of the mirror. As a result, the new mirror was able to be installed in the existing launchers without modifying the mirror mounts. This new design was also compatible with the higher magnetic fields on KSTAR.

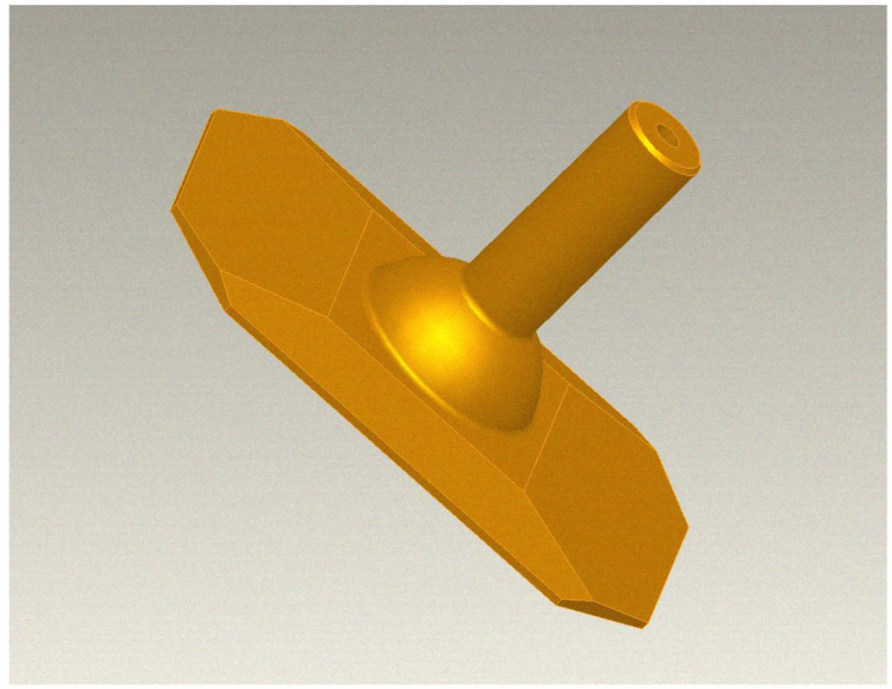

Figure 5. One-Piece Glidcop Fixed Mirror

The alloy chosen for the new fixed mirrors was Al-15 Glidcop [C15715]. This alumina dispersion strengthened copper alloy has good electrical conductivity and retains its strength at high temperatures. Plating of the front surface of these mirrors was not required. A modified Goodman diagram, with the peak operating stress superimposed, indicates that the fatigue life should be significantly more than the 4000 shots associated with a single run year of DIII-D.

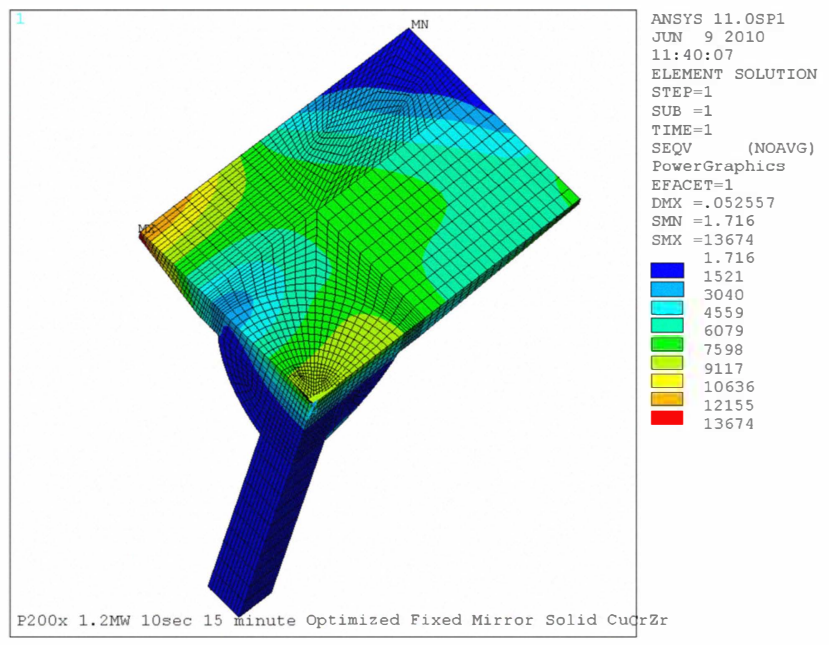

Figure 6. Fixed Mirror Stresses 


\section{STEERABle MirRors}

\section{A. P2001 Steerable Mirrors}

The original steerable mirrors for the DIII-D P2001 ECH launcher were made from a stainless steel block with copper bars inlaid longitudinally and brazed. The front, reflecting surface of the mirror was copper plated. The heat flux on the mirror surface diffuses through the copper bars rapidly, and then only has to penetrate the stainless steel in the thin direction. Initial thermal analysis indicated that the mirrors would remain two hundred degrees Celsius below the melting point of the Nioro braze alloy. This analysis, performed at the steepest angle of beam incidence, indicated a higher peak heat flux on these mirrors than that on the fixed mirrors. DIII-D typically operates with a shallow angle of beam incidence. In recent years, we had observed signs of thermal fatigue at the mirror surface. In view of the increased power load for the P2012 launcher, we redesigned the mirrors.

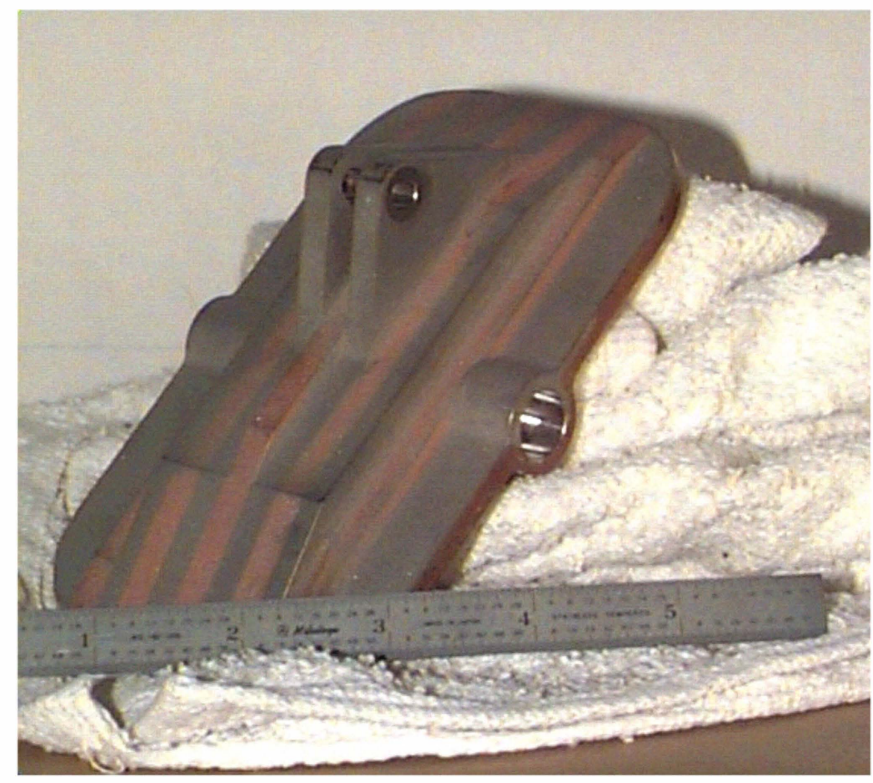

Figure 7. Figure 7. Rear view of P2001 Steerable Mirror

\section{B. P2012 Steerable Mirrors}

As with the design of the fixed mirrors, the objectives were to reduce the temperature excursions, reduce the thermal stresses, and reduce the effect of minor surface damage on mirror performance. These three goals were accomplished by making the new mirrors from a solid block of copper alloy, and then making a brazed assembly with inlaid stainless steel bars. Again using improved electromagnetic analysis, we were able to make the central area of the mirror from solid copper. The new mirrors were compatible with the existing steering pivots and steering mechanism on the DIII-D launchers, although they could not be used for KSTAR because of its higher toroidal field. Radiation provides adequate cooling for the new mirrors, and the maximum temperature is now roughly $550 \mathrm{C}-200 \mathrm{C}$ less than on the old design. The final version of these mirrors is being made from $\mathrm{C} 18150$ copper alloy. The maximum thermal strain in the front surface of these mirrors is $0.2 \%$. On the ITER design curve for $\mathrm{C} 18150$, this is below the design curve at 50,000 cycles.

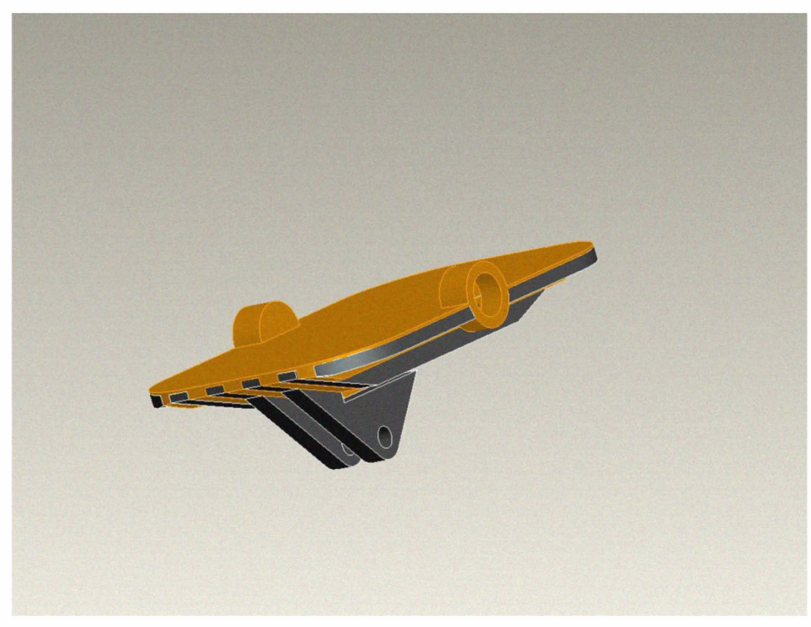

Figure 8. CAD model of P2012 Steerable Mirror

\section{CONCLUSIONS}

New fixed and steerable mirrors have been designed and fabricated for the DIII-D ECH launchers, with the goal of accommodating $1.5 \mathrm{MW}$ ECH pulses of 10 second durations. The new fixed mirrors have been installed in the three existing PPPL launchers at DIII-D, and will be installed on the P2012 launcher. One of these mirrors was also supplied with the KSTAR P2011 ECH launcher. The new steerable mirrors will be installed on the DIII-D P2012 ECH launcher, and retrofit on the existing three PPPL launchers.

\section{ACKNOWLEDGMENT}

This work is supported by the US DOE Contract No. DEAC02-09CH11466.

\section{REFERENCES}

[1] Prater, R. et al, "Applications of electron cyclotron waves in the DIII-d Tokamak," in AIP conference proceedings 1187, pp. 491-494

[2] Bae, Y. S. et al, "ECH pre-ionization and assisted startup in the fully superconducting KSTAR tokamak using second harmonic," Nuclear Fusion, Volume 49, Issue 2 (2009)

[3] Wagner, D. et al, "Recent Upgrades and Extensions of the ASDEX Upgrade ECRH System," in Journal of Infrared, Millimeter and Terahertz Waves, 32:274-282 (2011)

[4] Volpe, F. A. G. et al, "Advanced techniques for neoclassical tearing mode control in DIII-D," in Physics of Plasmas Vol 13 (2006), Number 5, p. 055501

[5] Lohr, J. et al, "The electron cyclotron resonant heating system on the DIII-D tokamak," in Fusion Science and Technology Volume 48 Number 2 (2005) pp. $1226-1237$

[6] Ellis, R. et al, "Design of the P2001 ECH launcher for DIII-D," in Proceedings - Symposium on Fusion Engineering (2002) pp. 133-136 